\title{
DECONTAMINATION INTERVENTIONS FOR THE REUSE OF SURGICAL MASK PERSONAL PROTECTIVE EQUIPMENT: A SYSTEMATIC REVIEW
}

David J Zorko, MD1; Shira Gertsman, BSc2; Katie O’Hearn, MSc2; Nicholas Timmerman, MD3;

Nasser Ambu-Ali, MD1; Tri Dinh, BSc4; Margaret Sampson, MLIS PhDs; Lindsey Sikora, MISt6; James Dayre McNally, MSc PhD2,7; and Karen Choong, MBBCh MSc1,8,9

1 Department of Pediatrics, McMaster University, Hamilton, Ontario, Canada

${ }_{2}$ Children's Hospital of Eastern Ontario Research Institute, Ottawa, Ontario, Canada

3 Department of Anesthesia, McMaster University, Hamilton, Ontario, Canada

4 Michael G. DeGroote School of Medicine, McMaster University, Hamilton, Ontario, Canada

5 Library Services, Children's Hospital of Eastern Ontario, Ottawa, Ontario, Canada

${ }_{6}$ Health Sciences Library, University of Ottawa, Ottawa, Ontario, Canada

7 Department of Pediatrics, Children's Hospital of Eastern Ontario, Ottawa, Ontario, Canada

8 Department of Critical Care, McMaster University, Hamilton, Ontario, Canada

${ }_{9}$ Department of Health Research Methods, Evidence, and Impact, McMaster University, Hamilton, Ontario, Canada

Corresponding Author: Dr. Karen Choong (ORCID ID 0000-0002-4608-4508)

Department of Pediatric Critical Care, McMaster University, Room 3E20

1280 Main Street West, Hamilton, Ontario, Canada, L8N $3 Z 5$

E-mail: choongk@mcmaster.ca

Running Title: Surgical Mask Decontamination 


\section{ABSTRACT}

Background: The high demand for personal protective equipment (PPE) during the novel coronavirus outbreak has created global shortages and prompted the need to develop strategies to conserve supply. Surgical mask PPE have a broad application of use in a pandemic setting, but little is known regarding decontamination interventions to allow for their reuse. Objective: Identify and synthesize data from original published studies evaluating interventions to decontaminate surgical masks for the purpose of reuse. Methods: We searched MEDLINE, Embase, CENTRAL, Global Health, the WHO COVID-19 database, Google Scholar, DisasterLit, preprint servers, and prominent journals from inception to April 8, 2020 for prospective original research on decontamination interventions for surgical mask PPE. Citation screening was conducted independently in duplicate. Study characteristics, interventions, and outcomes were extracted from included studies by two independent reviewers. Outcomes of interest included impact of decontamination interventions on surgical mask performance and germicidal effects. Results: Seven studies met eligibility criteria: one evaluated the effects of heat and chemical decontamination interventions applied after mask use on mask performance, and six evaluated interventions applied prior to mask use to enhance antimicrobial properties and/or mask performance. Mask performance and germicidal effects were both evaluated in heterogenous test conditions across a variety of mask samples (whole masks and pieces or individual mask layers).

Safety outcomes were infrequently evaluated. Mask performance was best preserved with dry heat decontamination. Germicidal effects were best in salt-, N-halamine- and nanoparticle-coated masks. Conclusion: There is limited evidence on the safety or efficacy of surgical mask decontamination. Given the heterogenous methods used in the studies to date, we are unable to draw conclusions on the most appropriate, safest intervention(s) for decontaminating surgical masks for the purpose of reuse. 
Keywords: COVID-19, SARS-CoV-2, PPE, decontamination, masks, surgical masks, systematic review

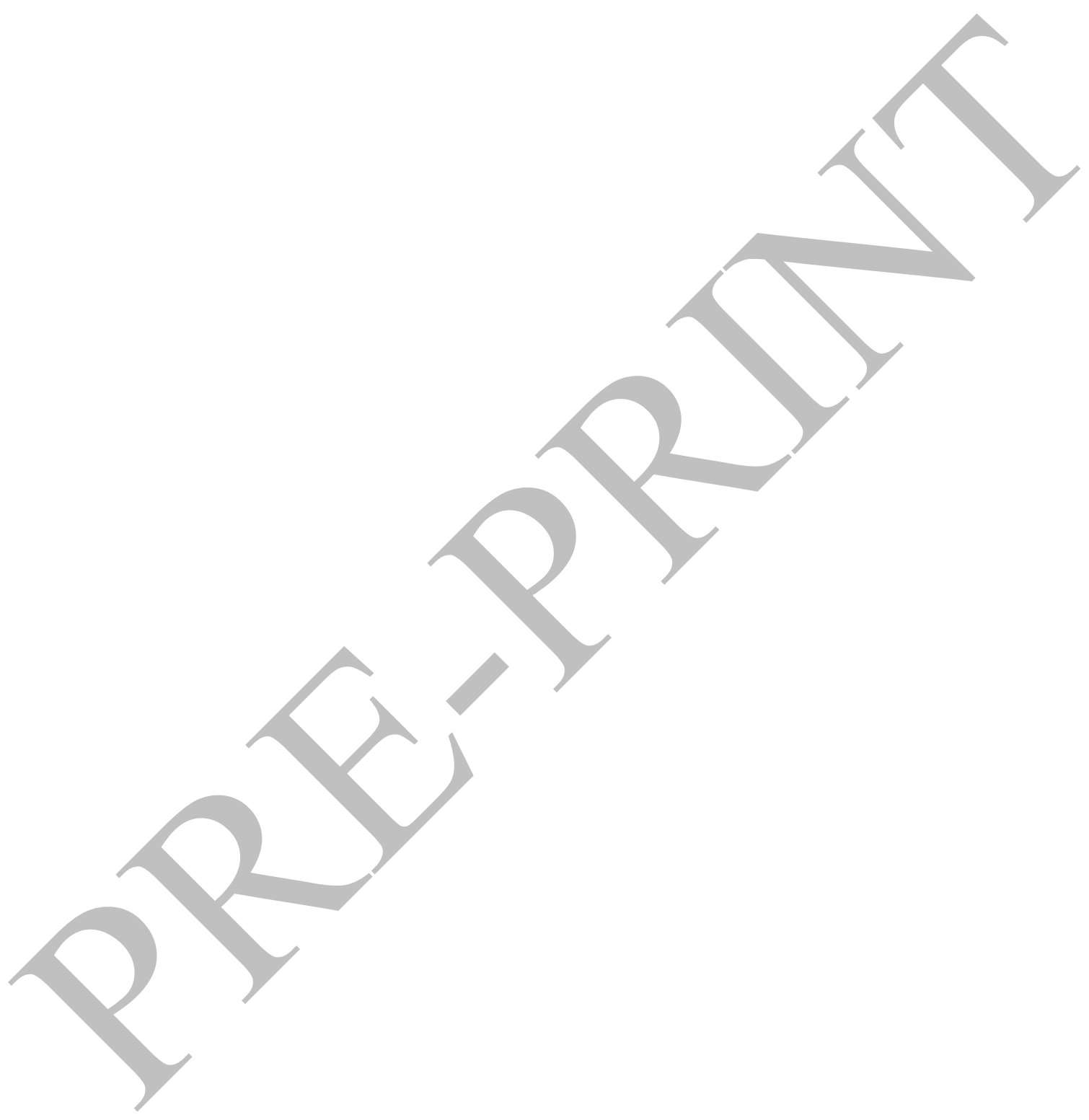




\section{Background \& Rationale}

As the global spread of novel coronavirus (SARS-CoV-2) continues to escalate, so has the demand for personal protective equipment (PPE), creating global shortages in the supply of N95 filtering face respirators (FFRs) and surgical masks. N95 FFRs are recommended by the World Health Organization (WHO) and the Centers for Disease Control and Prevention (CDC) for use by healthcare providers (HCPs) caring for coronavirus disease (COVID-19) patients requiring airborne precautions and during aerosol-generating procedures [1, 2]. Therefore, N95 FFRs are most commonly needed for HCPs in acute care and inpatient settings.

In contrast, surgical masks are recommended for use by HCPs to protect against the risk of droplet transmission in a broader range of inpatient healthcare settings, as well as outpatient settings (e.g. COVID-19 assessment centres, long-term care facilities, and community care settings) [2-4]. Surgical masks are also recommended for use by patients with suspected or confirmed COVID-19 to prevent potential spread in a variety of healthcare settings $[2,4]$. PPE recommendations are dynamic and continue to broaden in response to the spread and our growing understanding of SARS-CoV-2 [4, 5]. Several institutions now recommend that everyone entering the hospital setting wear a surgical mask, regardless of whether they will have patient contact [6]. Furthermore, as the supply of N95 FFRs is threatened, healthcare professionals may resort to use of surgical masks for airborne precautions $[3,7-9]$.

These practices have created an unprecedented demand for surgical masks; unfortunately, the capacity for surge production of PPE is not sustainable in the long-term [7-9]. As most facemask PPE are designed for single-use, mask rationing and conservation is now a top priority globally [10]. Reusing these disposable masks is now suggested as a crisis capacity strategy to conserve available supplies during a pandemic [2, 10-12]. Subsequently, much attention has now turned to reprocessing and decontamination of facemask PPE. Several strategies such as 
ultraviolet germicidal irradiation (UVGI), chemical disinfectants (e.g. hydrogen peroxide vapour, ethanol), and microwave- and heat-based methods have been evaluated; however, most of this literature has focussed on the decontamination of N95 FFRs [13-15].

The evidence on the efficacy and safety of decontamination and reuse of surgical masks is unclear. The objective of this systematic review was to evaluate and synthesize the evidence on decontamination or sterilization interventions for the purpose of surgical mask PPE reuse.

\section{Methods}

This systematic review protocol was designed a priori, registered on PROSPERO (April 15th, 2020; CRD42020178290), and uploaded as a pre-print on Open Science Framework (April 8th, 2020; https://osf.io/8wt37/) [16]. The reporting of this systematic review is in accordance with the Preferred Reporting Items for Systematic Reviews and Meta-Analyses (PRISMA) statement (Appendix A) [17].

\section{Eligibility Criteria}

Studies published in English or French were eligible for inclusion if the following criteria were met: 1) The study was original research, including systematic reviews;2) The study evaluated surgical facemask PPE or their components; 3 ) The study evaluated any intervention(s) to decontaminate, sterilize or treat surgical masks (applied either before or after their use) for the purposes of reuse as PPE; 4) At least one of the following efficacy or safety outcomes of interest was reported: a) mask filtration performance (i.e. filtration efficiency and airflow resistance); b) reduction in pathogen load; c) clinical infection rates following use of the decontaminated masks; d) changes in physical state (i.e. mask appearance or physical degradation); e) adverse effects experienced by the wearer (e.g. skin irritation); or f) feasibility of the intervention (e.g. time, cost, 
resource utilization). We excluded editorials, case reports, narrative reviews, study protocols, clinical practice guidelines, grey literature, book chapters, and patents.

\section{Database Search \& Study Selection}

Two health sciences librarians (LS, MS) searched the following electronic databases from their dates of inception to April 8th, 2020: Medline and Medline in Process via OVID, Embase Classic + Embase via OVID, Cochrane CENTRAL (February 2020 issue) and Global Health via CAB Direct. A search strategy was developed in Medline, and then translated into the other databases, as appropriate (Appendix B). Language was restricted to English or French, with no other publication restrictions.

Three journals were also hand-searched, as they were particularly relevant to the review but are not indexed in any of the electronic databases: Journal of the International Society for Respiratory Protection, Aerosol Science and Technology, and the Journal of Engineered Fibers and Fabrics. A search of Google Scholar (April 8th, 2020) through Publish or Perish was screened until 50 consecutive apparently irrelevant citations were found. Results to that point were saved as an RIS file and edited to remove patents, reports and books. The WHO database on COVID-19 as of April 7th, 2020 was downloaded and searched within Reference Manager. Disaster Lit: Database for Disaster Medicine and Public Health, MedRxiv, and OSF Preprints were searched on April 8th, 2020 and citations pertaining to decontamination were selected and downloaded. All citations were entered into an Endnote file and duplicates were removed.

\section{Citation Screening \& Data Extraction}

Citations were uploaded to insightScope (www.insightscope.ca) for title/abstract screening and full text review. Citation screening for title/abstract and full text review stages was conducted 
independently and in duplicate by a team of 11 reviewers recruited from McMaster University, the University of Ottawa, and the University of Manitoba. Prior to gaining access to the full set of citations, each reviewer read the systematic review protocol and was required to achieve a sensitivity of at least $80 \%$ when screening a test set of 50 citations (containing 5 true positives and 45 true negatives). Reviewers achieving less than $80 \%$ sensitivity on the test set were provided with additional training prior to gaining access to the full set of citations. At both title/abstract and full text review, citations were excluded only if both reviewers agreed to exclude; disagreements were reviewed and resolved by the study leads where necessary (DZ, $\mathrm{KC}$ ). Upon completion of full text review, the study co-lead(DZ) reviewed all retained citations to identify potential duplicates and confirm eligibility. The reference lists of all citations included for full text review were searched for potential eligible citations that may have evaded the initial database search.

Data were collected using electronic data extraction forms (Microsoft Excel) modified from previous systematic reviews $[13,14]$ for this protocol and piloted by two investigators (DZ, SG) on two eligible studies. Data was extracted from the full text publication and any related publications, referenced published protocols, or supplementary materials. Data extraction was completed in duplicate by two independent reviewers. Where necessary, graphical data was extracted using SourceForge Plot Digitizer (http://plotdigitizer.sourceforge.net) and checked by the second reviewer for accuracy. Disagreements were resolved by reviewer consensus and discussion with the study leads (DZ, KC), where necessary.

\section{Risk of Bias Assessment}

We planned to use recommended risk of bias tools where appropriate [18]; however, in the absence of a standard risk of bias tool for laboratory studies, we applied objective assessment 
criteria developed for this purpose [15]. Risk of bias was assessed by two reviewers independently and in duplicate at the study level by outcome in the following domains: study design, methodological consistency, population heterogeneity, sampling bias, outcome evaluation, and selective reporting (Appendix C).

\section{Outcomes}

The primary outcome for this study was efficacy and safety of the decontamination intervention, as determined by any of the following: mask performance (filtration efficiency [FE] and airflow resistance); reduction in pathogen load; clinical infection rates following use of treated masks; mask appearance or physical degradation; or adverse effects experienced by the wearer (e.g. skin irritation). FE refers to the percentage of particles filtered at a specified particle size, which may be $0.1 \mu \mathrm{m}$ or $3 \mu \mathrm{m}$ depending on the testing agent and standard used [19]. For this systematic review, we converted study results reporting percentage particle penetration, to FE units (i.e. $\mathrm{FE} \%=100-$ particle penetration) for comparability [19]. Airflow resistance is measured as the pressure drop across the mask, quantifying initial resistance to airflow in millimetres of water column height pressure per square centimeter $\left(\mathrm{mmH}_{2} \mathrm{O} / \mathrm{cm}_{2}\right)$ [20]. Greater airflow resistance indicates less breathability of the mask. Reduction in pathogen load/titers was reported as the log reduction from a time zero post-inoculation to a subsequently measured time point. If log reductions were not reported by the study, we calculated them using the pathogen load data provided (e.g. colony counts), where possible. A $\log$ reduction $\geq 3$ was used as a reference indicating good germicidal effect [21]. The secondary outcome was feasibility of the intervention, such as the time, cost and resources required to implement the intervention. 
Where series of results were presented for multiple experimental conditions, we report the summary of results conducted at the harshest testing conditions, to allow a conservative interpretation of the outcomes evaluated [19].

\section{Statistical Analysis}

Primary outcome data was analysed descriptively and presented using absolute values and as a percent change where possible. No three studies evaluated the same intervention, nor applied similar test agents or conditions when evaluating outcomes. This precluded pooling of data and planned quantitative analysis of outcomes [16]; therefore, selected results from included studies were summarized descriptively.

\section{Results}

\section{Study Identification}

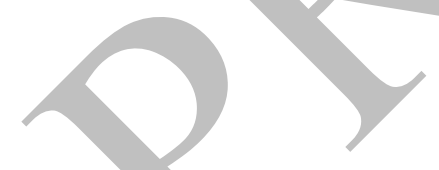

Nine of 11 reviewers achieved the $80 \%$ sensitivity threshold on the test set. The two reviewers who did not achieve the $80 \%$ threshold were provided additional training regarding the screening protocol prior to citation screening. The review team achieved kappa values of 0.44 and 045 for title/abstract and full text screening respectively. Study leads resolved conflicts in $3.0 \%$ title/abstract and $12.1 \%$ full text screening citations.

A total of 2191 records were identified through the initial database search, of which 1874 unique citations were reviewed and 33 full-texts were retained and assessed for eligibility. Twenty-six full texts were excluded for not meeting eligibility criteria, leaving seven unique studies for inclusion in our analysis (PRISMA diagram, Figure 1). No additional citations were identified on review of reference lists. 


\section{Study Characteristics}

Characteristics of included studies are summarized in Table 1. Only one of the seven included studies specifically evaluated decontamination interventions applied after surgical mask wear, for the purpose of reuse [22]. The remaining six studies evaluated interventions applied to masks or mask components prior to use to enhance antimicrobial properties and/or FE for potential reuse or extended use (i.e. pre-contamination interventions) [23-28]. Interventions in these studied were tested on coated and control (uncoated) whole masks, pieces of whole masks (referred hereafter as mask pieces) or pieces of individual mask layers (referred hereafter as mask layer pieces). Risk of bias assessments for the included studies are described in Appendix D.

\section{Decontamination Interventions for Surgical Mask Reuse}

Lin et al. [22] evaluated five decontamination interventions on two surgical mask types commonly used in Taiwanese hospitals (gauze double-layer electret masks and Oimo spunlace non-woven masks; models unspecified): dry heat (via rice cooker), high-pressure moist heat (i.e. autoclave), and three chemical agents ( $70 \%$ ethanol, $100 \%$ isopropanol, and $0.5 \%$ sodium hypochlorite [i.e. bleach])[22]. The methods and findings of this study are summarized in Table 2. Mask pieces were assessed for FE, airflow resistance, and physical characteristics after each interyention was applied. FE was presented graphically for a range of particle sizes $(0.0146 \mu \mathrm{m}$ to $0.594 \mu \mathrm{m}$ ); we summarized the results for FE at $0.1 \mu \mathrm{m}$, a standard particle size for viral FE testing [19]. At baseline, gauze and spunlace masks had FEs of approximately $87 \%$ and $45 \%$, respectively. FE for both masks decreased after each of the five decontamination interventions, but the smallest change was observed following dry heat treatment of the gauze mask (absolute reduction of $1.3 \% \mathrm{FE})$. Moist heat and chemical decontamination interventions all resulted in greater FE decreases in both mask types, with absolute reductions ranging from $12 \%$ to $36 \%$. 
Bleach was the most damaging method, resulting in an absolute FE reduction of $15.3 \%$ in the spunlace mask and destruction of the gauze mask.

Airflow resistance was assessed at a flow rate of $5.95 \mathrm{~L} / \mathrm{min}$ [22]. Statistically significant changes in pressure drop were reported following all decontamination interventions, except for dry heat and ethanol on gauze mask pieces. Airflow resistance results were not reported for bleach on gauze masks (mask destroyed), or isopropanol for either mask type. Physical appearance changes were reported only for gauze masks; the autoclave deformed and caused observable folds in the mask filter, and bleach destroyed the mask. Physical characteristics following decontamination with other interventions, or in spunlace masks, were not reported. This study did not assess the germicidal effects of any of the five decontamination methods.

\section{Pre-Contamination Interventions}

Six studies evaluated five unique pre-contamination methods applied prior to mask use: four were antimicrobial interventions. (nanoparticle emulsion [24, 25], quaternary ammonium agent [28], monochlorinated $\mathrm{N}$-halamine [23], sodium chloride [ $\mathrm{NaCl}]$ salt [26]), and one was a fluorochemical repellent [27] (Table 1).

These interventions have a variety of proposed mechanisms of action. Quan et al. [26] evaluated multiple concentrations of "salt-functionalized filters"- $\mathrm{a} \mathrm{NaCl}$ salt coating applied to the polypropylene middle layer of a three-ply surgical mask, which inactivates viruses by hyperosmotic stress upon the viral envelope. Tseng et al. [28] tested a quaternary ammonium agent (Goldshield 5 [GS5], AP Goldshield LLC, USA), a disinfectant commonly used in medical, commercial and household settings. Demir et al. [23] applied a monochlorinated N-halamine coating onto the polypropylene layer of a surgical mask, which inactivates Gram-negative and Gram-positive bacteria through direct transfer of oxidative halogens to the cell membrane. Li et 
al. $[24,25]$ conducted two studies evaluating a silver nitrate and titanium dioxide nanoparticle emulsion coating of the outer layer of a surgical mask. Silver inactivates bacteria by interacting with the thiol groups of proteins and enzymes, and titanium oxide creates microbicidal hydroxyl radicles [24]. Finally, Shen et al. [27] applied a fluorochemical finish (Zonyl® PPR Protector, Ciba Specialty Chemicals, Switzerland) to the outer layer of surgical masks to enhance resistance to fluid penetration.

\section{Filtration Efficiency and Airflow Resistance Following Pre-Contamination}

Five studies evaluated the effects of their intervention on FE, airflow resistance, or both, applying

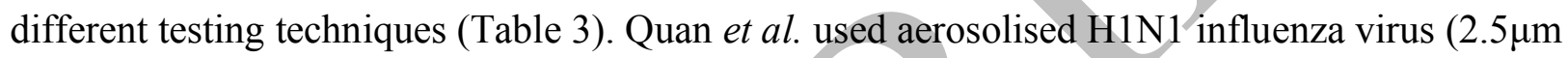
to $4 \mu \mathrm{m}$ particle diameter) to evaluate $\mathrm{FE}$ on polypropylene mask filter pieces and found that increasing concentrations of the salt coating increased filter EE $(54.4 \%, 69 \%$ and $83.9 \%$ for 3,11 and $19 \mathrm{mg} \mathrm{NaCl} / \mathrm{cm} 2$, respectively) [26]. Airflow resistance was not assessed in this study. Tseng et al. evaluated FE of GS5-coated mask layer pieces using aerosolized bacteria $(0.5 \mu \mathrm{m}$ to $2.1 \mu \mathrm{m}$ particle diameter) and GS5-coated masks using $\mathrm{NaCl}(0.075 \mu \mathrm{m}$ particle diameter), respectively, compared to uncoated controls. They found no statistically significant change in FE in the GS5coated masks or mask layer pieces ( $0.6 \%$ to $1.8 \% \mathrm{FE}$ increase in polypropylene filter layer, $1.8 \%$ FE reduction in mask; $p=N S$ ) [28]. Airflow resistance was also not significantly affected (pressure drop change of $1.0 \mathrm{mmH}_{2} \mathrm{O} ; \mathrm{p}=\mathrm{NS}$ ). Demir et al. only assessed airflow resistance and found similar results in N-halamine-treated polypropylene filters compared to untreated filters $(26.7 \mathrm{ml} / \mathrm{s} / \mathrm{cm} 2 ; \mathrm{p}=\mathrm{NS})[23]$.

Li et al. [25] used a potassium-fluorescein solution (particle size not reported) to test nanoparticle-coated full masks and expressed their results in FE as: 1) the percentage potassium content of each mask layer relative to the potassium content of the whole mask; and 2) a seven 
point scale rating fluorescent stains on the face of mask users. They found that the percentage potassium content of each mask layer was similar compared to uncoated masks $(+2 \%,-3 \%$ and $+1.5 \%$ absolute difference from control for outer, middle and interior mask layers, respectively), and similar ratings of fluorescent stains. Airflow resistance was non-significantly increased $(+1.4$ $\left.\mathrm{ml} / \mathrm{s} / \mathrm{cm}_{2} ; \mathrm{p}=\mathrm{NS}\right)$. Shen et al. [27] used an aerosolized pathogen simulant (1.0 $\mu \mathrm{m}$ particle diameter) and also quantified $\mathrm{FE}$ as the percentage particle content of each mask layer to the particle content of the whole mask. They reported significant decreases in particle content on the repellant-coated outer mask layer coated with repellant $(\mathrm{p}<0.0001)$, but no changes to particle content on mask layers distal to the filter layer (suggesting no changes to FE of the mask as a whole). Airflow resistance was not assessed.

\section{Germicidal Effects of Pre-Contamination Interventions}

Four studies evaluated germicidal effects using a variety of aerosolized pathogens $[23,26,28]$ or bacterial suspensions $[23,24]$, and assessed the change in pathogen load upon the mask or mask component(s) tested after different incubation periods (Table 3). All three tested concentrations of salt-treated polypropylene mask filters exhibited reductions in bacterial colony counts at 5 and 15 minutes post-incubation, and were below detection limit (BDL) by 60 minutes [26].

Germicidal effect was strongest in the $19 \mathrm{mg} \mathrm{NaCl} / \mathrm{cm} 2$ salt-coated mask filter (log reduction $\geq 3$ at 5 and 15 minutes, and BDL at 60 minutes). Colony counts demonstrated a greater than $3 \log$ reduction in $\mathrm{N}$-halamine-coated mask filters when evaluated 10 minutes after a 3-hour bacterial aerosol test [23], and in the nanoparticle-coated mask outer layer at 24 hours post-incubation with bacterial suspensions [24]. GS5-coated mask layers did not appear to achieve adequate reduction in pathogen load (log reduction $\leq 1$ in all experiments) [28]. 
In vivo infection was evaluated by Quan et al., where 16-day survival of mice was assessed following exposure to aerosolized influenza viruses through a salt-treated mask filter barrier compared to an uncoated filter barrier. Mice sheltered by uncoated filter barriers were euthanized after losing more than $25 \%$ of their body weight (a surrogate outcome for mortality) by day 16 , while none of the mice sheltered by the salt-coated mask filter irrespective of salt coating concentration, met this endpoint (i.e. 100\% survival rate) [26]. Lung viral titers were also assessed; titers were lower, but still detectable, in lungs of mice sheltered by the salt-coated mask filters compared to those sheltered by uncoated filter barriers.

\section{Physical Characteristics and Adverse Events following Pre-Contamination}

Two studies assessed physical characteristics of nanoparticle-coated masks and/or adverse effects after their use in human subjects [24, 25]. A survey of blinded users of nanoparticle-coated and uncoated masks demonstrated no differences in perceived heat, humidity, odour, breathability, itchiness, or user comfort between the two groups ( $p=$ NS in each survey domain) [25]. Symptom inquiry and physical examination of the facial skin in users of nanoparticle-coated and uncoated masks demonstrated no signs or symptoms of skin irritation between the two groups [24].

\section{Discussion}

This systematic review on the evidence for decontamination interventions for surgical mask PPE reveals the following key findings. First, there is a paucity of research evaluating decontamination interventions on surgical masks; there is only one study evaluating methods intended for decontaminating masks following use, and six studies evaluating pre-contamination methods to enhance the antimicrobial and/or filtration performance of surgical masks. Second, included studies evaluated different interventions, using heterogenous and non-standardized test 
conditions, and assessed a variety of outcomes using inconsistent methods. This limits our ability to make comparisons between interventions and conclusions regarding their efficacy or safety. Furthermore, no studies provided explicit information on the feasibility, resource requirements, or applicability of these decontamination methods in institutional or hospital settings, making it challenging to determine if any of the interventions evaluated could be easily implemented during situations of high PPE demand.

Each decontamination intervention used in the included studies is founded in evidencebased scientific rationale. Heat is known to inactivate pathogens, including coronaviruses [2931]; moist heat is a standard intervention for sterilization in healthcare facilities [32, 33]. The chemical disinfectants evaluated in the included studies are also widely used in household, commercial, healthcare, and food industry settings [32,34]. Based on the limited evidence in this review, dry heat may alter surgical mask performance less than high-pressure moist heat or chemical interventions; however, the germicidal effect of dry heat in surgical masks is unclear. Bleach is not a safe method of decontaminating surgical masks; surgical mask performance is significantly altered and safety data from N95 FFR studies suggest potential health risks associated with off-gassing [15]. With respect to pre-contamination interventions, masks coated with salt films, GS5, nanoparticle emulsion and N-halamines were reported not to significantly alter mask performance. Of the interventions tested, $\mathrm{N}$-halamine and nanoparticle emulsion showed strong germicidal effects in masks ( $\log$ reductions $\geq 3$ ), which is consistent with their application in medical devices [35], and food and water treatment [36]. Salt films also demonstrated strong germicidal effects in masks, but their application has been experimental to date [26]. An important consideration is that pathogen load was evaluated at different postinoculation incubation time points in each study (i.e. five minutes to 24 hours) and it is wellestablished that viral load reductions can occur by virtue of time [37]. 
Ideal PPE decontamination methods should not only demonstrate effective reductions in pathogen burden, but also preserve the structural and functional integrity of the mask without causing any residual chemical hazard to the wearer [38]. The evidence that we have collated in this systematic review is therefore important and essential. However, we advise caution when interpreting some of the results of included studies for the following reasons: 1) some of the mask types used in these experiments appear to have baseline filtration efficiencies below reference standards which may have affected the results observed [19]; 2) experiments and test conditions applied to mask pieces or individual layers cannot necessarily be extrapolated to whole masks; and 3) the testing conditions and methods of reporting results were heterogenous. In contrast to N95 FFRs, surgical masks are not certified under the National Institute for Occupational Safety and Health regulations. The Food and Drug Administration recommends that several standards (ASTM F2101, ASTM 2299, Mil-M369454C, or modified Greene and Vesley method) may be applied to surgical masks, complicating the evaluation of mask performance in this study [19].

This systematic review reveals that the body of evidence on decontamination interventions for surgical masks is scant compared to N95 FFRs. Three recent systematic reviews have revealed 22 unique studies evaluating interventions such as microwave irradiation, heat, chemical disinfectants, and ultraviolet germicidal irradiation (UVGI) for decontamination of N95 FFRs [13-15]. UVGI and yaporous hydrogen peroxide were the most commonly evaluated and showed favourable evidence for germicidal effects without significant harm to mask performance; however, we were not able to find any publications evaluating these methods in surgical masks. The lack of research on surgical masks may stem from assumptions that methods effective in N95 FFRs can be extrapolated to surgical masks; some institutions are already applying the same decontamination methods to both N95 FFR as well as surgical masks [39]. Considering this systematic review demonstrates that different mask types can perform 
differently after decontamination, and that surgical masks and N95 FFRs perform differently with aerosol challenges $[22,40]$, we cannot conclude that the same decontamination methods can be effectively or safely applied to all mask PPE types. Furthermore, there is limited data evaluating the effectiveness of any decontamination intervention against SARS-CoV-2 on PPE [39, 41], although more studies are underway. Independent research on surgical masks is therefore critical in order to inform clinicians, infection control experts, and public health administrators on how best to advise safe decontamination and reuse practices.

\section{Strengths \& Limitations}

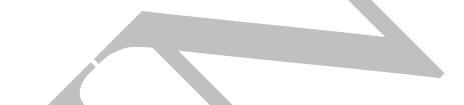

Our systematic review has several important strengths. To our knowledge, this is the first systematic review of decontamination interventions for the reuse of surgical mask PPE and provides important information describing the nature of interventions and outcomes evaluated to date. Our review highlights the variability in methodology and reporting of evaluated outcomes. As a result, we identified the following core outcomes to consider when conducting research in this field, to encourage more consistency in methodology and transparency of reporting: mask performance (FE, airflow resistance), decontamination effects (germicidal effects, in vivo infection rates), physical characteristics of decontaminated masks, adverse effects to mask users, and feasibility of the interyention. We also developed a systematic tool with which to assess risk of bias in this body of literature.

Our review also has limitations. We were unable to conduct any meta-analyses due to the paucity of studies and their heterogeneous methodologies and outcome assessments. Outcomes described this systematic review required summarizing study results from multiple experiments; we rationalized the selective reporting of results in our methods to encourage conservative interpretation of the findings. Given the rapidly evolving landscape of PPE literature during the 
SARS-CoV-2 pandemic, we plan to update this systematic review at regular intervals for new relevant evidence as it becomes available (i.e. living systematic review) [42].

\section{Conclusion}

There is inadequate evidence on the safety or efficacy of any decontamination intervention for extended use or reuse of surgical masks in the clinical setting. Further research should therefore be conducted specifically in surgical masks, that include decontamination interventions demonstrating promise in N95 FFRs (e.g. UVGI, vaporized hydrogen peroxide). To ensure the safety of HCPs and all end users, the same rigorous standard of research should be applied to surgical masks as with N95 FFRs, given its much broader applications as PPE. We recommend that future studies in this area consider applying core outcomes and test conditions that are in accordance with acceptable industry standards in their design, to enable transparency of reporting, interpretation of result in context of a particular setting or pathogen in question, and to enable future comparisons of efficacy between interventions. 
Abbreviations: BDL, below detection limit; CDC, Centers for Disease Control and Prevention; COVID-19, coronavirus disease; FE, filtration efficiency; FFR, filtering face respirators; GS5, Goldshield 5 quaternary ammonium agent; $\mathrm{HCP}$, healthcare provider; $\mathrm{NaCl}$, sodium chloride; NS, not statistically significant; PPE, personal protective equipment; PRISMA, Preferred Reporting Items for Systematic Reviews and Meta-Analyses; SARS-CoV-2, novel coronavirus; UVGI, ultraviolet germicidal irradiation; WHO, World Health Organization.

Acknowledgements: The authors are grateful to the following people for their contributions to citation screening (in alphabetical order): Anirudh Agarwal, Kwame Agyei, Jess Gibson, Hendrick Guerra, Ronish Gupta, Manvinder Kaur, Rhiannon Ng, Emiliyan Staykov.

Conflict of Interest: None to declare.

Source of Funding: This research did not receive any specific funding. 


\section{References}

1. Centers for Disease Control and Prevention. Interim Infection Prevention and Control Recommendations for Patients with Suspected or Confirmed Coronavirus Disease 2019 (COVID-19) in Healthcare Settings. 2020; https://www.cdc.gov/coronavirus/2019ncov/hcp/infection-control-recommendations.html. [Accessed April 18, 2020]

2. World Health Organization. Rational use of personal protective equipment for coronavirus disease (COVID-19) and considerations during severe shortages: interim guidance, 6 April 2020. Geneva: World Health Organization; 2020.

3. MacIntyre CR, Chughtai AA. Facemasks for the prevention of infection in healthcare and community settings. BMJ. 2015;350:h694.

4. World Health Organization. Advice on the use of masks in the context of COVID-19: interim guidance, 6 April 2020. Geneva: World Health Organization; 2020.

5. Feng S, Shen C, Xia N, et al. Rational use of face masks in the COVID-19 pandemic. The Lancet Respiratory Medicine. 2020.

6. Klompas M, Morris CA, Sinclair J, et al. Universal masking in hospitals in the Covid-19 era. New England Journal of Medicine. 2020.

7. Carias C, Rainisch G, Shankar M, et al. Potential Demand for Respirators and Surgical Masks During a Hypothetical Influenza Pandemic in the United States. Clinical Infectious Diseases. 2015;60(suppl_1):S42-S51.

8. Patel A, D'Alessandro MM, Ireland KJ, et al. Personal Protective Equipment Supply Chain: Lessons Learned from Recent Public Health Emergency Responses. Health Security. 2017;15(3):244-252.

9. Srinivasan A, Jernign DB, Liedtke L, et al. Hospital Preparedness for Severe Acute Respiratory Syndrome in the United States: Views from a National Survey of Infectious Diseases Consultants. Clinical Infectious Diseases. 2004;39(2):272-274.

10. Centers for Disease Control and Prevention. Strategies for Optimizing the Supply of Facemasks. 2020; https:/www.cdc.gov/coronavirus/2019-ncov/hcp/ppe-strategy/facemasks.html. [Accessed April 18, 2020]

11. Livingston E, Desai A, Berkwits M. Sourcing Personal Protective Equipment During the COVID-19 Pandemic. JAMA. 2020.

12. Centers for Disease Control and Prevention. Decontamination and Reuse of Filtering Facepiece Respirators. 2020; https://www.cdc.gov/coronavirus/2019-ncov/hcp/ppestrategy/decontamination-reuse-respirators.html. [Accessed April 18, 2020]

13. Gertsman S, Agarwal A, O'Hearn K, et al. Microwave-and Heat-Based Decontamination of N95 Filtering Facepiece Respirators (FFR): A Systematic Review. OSF Preprints. 2020. 
14. O'Hearn K, Gertsman S, Sampson M, et al. Decontaminating N95 masks with Ultraviolet Germicidal Irradiation (UVGI) does not impair mask efficacy and safety: A Systematic Review. OSF Preprints. 2020.

15. O'Hearn K, Gertsman S, Webster RJ, et al. Efficacy and Safety of Disinfectants for Decontamination of N95 and SN95 Filtering Facepiece Respirators: A Systematic Review. OSF Preprints. 2020.

16. Zorko D, Choong K, McNally D, et al. Decontamination Interventions for the Reuse of Surgical Mask Personal Protective Equipment (PPE): A Protocol for a Systematic Review. OSF Preprints. 2020.

17. Liberati A, Altman DG, Tetzlaff J, et al. The PRISMA Statement for Reporting Systematic Reviews and Meta-Analyses of Studies That Evaluate Health Care Interventions: Explanation and Elaboration. PLOS Medicine. 2009;6(7):e1000100.

18. Page MJ, McKenzie JE, Higgins JPT. Tools for assessing risk of reporting biases in studies and syntheses of studies: a systematic review. BMJ Open. 2018;8(3):e019703.

19. Rengasamy S, Shaffer R, Williams B, Smit S. A comparison of facemask and respirator filtration test methods. Journal of occupational and environmental hygiene. 2017;14(2):92-103.

20. American Society for Testing and Materials (ASTM) F2100-04. Standard specification for performance of materials used in medical face masks.

21. Fisher EM, Noti JD, Lindsley WG, et al. Validation and application of models to predict facemask influenza contamination in healthcare settings. Risk analysis : an official publication of the Society for Risk Analysis. 2014;34(8):1423-1434.

22. Lin, T.-H., et al., Filter quality of electret masks in filtering 14.6-594 nm aerosol particles: Effects of five decontamination methods. PloS one, 2017. 12(10). Lin T-H, Chen C-C, Huang S-H, et al. Filter quality of electret masks in filtering 14.6-594 nm aerosol particles: Effects of five decontamination methods. PloS One. 2017;12(10).

23. Demir B, Cerkez I, Worley S, et al. N-halamine-modified antimicrobial polypropylene nonwoven fabrics for use against airborne bacteria. ACS Applied Materials \& Interfaces. 2015;7(3):1752-1757.

24. Li Y, Leung P, Yao L, et al. Antimicrobial effect of surgical masks coated with nanoparticles. Journal of Hospital Infection. 2006;62(1):58-63.

25. Li Y, Wong $\mathrm{T}$, Chung, et al. In vivo protective performance of $\mathrm{N} 95$ respirator and surgical facemask. American journal of industrial medicine. 2006;49(12):1056-1065.

26. Quan F-S, Rubino I, Lee S-H, et al. Universal and reusable virus deactivation system for respiratory protection. Scientific Reports. 2017;7(1):1-10.

27. Shen H, Leonas KK. Study of Repellent Finish Of Filtration Ability Of Surgical Face Masks. International Nonwovens Journal. 2005(4):1558925005os-1551400403. 
28. Tseng C-C, Pan Z-M, Chang C-H. Application of a quaternary ammonium agent on surgical face masks before use for pre-decontamination of nosocomial infection-related bioaerosols. Aerosol Science and Technology. 2016;50(3):199-210.

29. American Society for Testing and Materials (ASTM) F2299-03. Test method for determining the initial efficiency of materials used in medical face masks to penetration by particulates using latex spheres.

30. Leclercq I, Batejat C, Burguière AM, Manuguerra JC. Heat inactivation of the Middle East respiratory syndrome coronavirus. Influenza and other respiratory viruses. 2014;8(5):585-586.

31. Rabenau H, Cinatl J, Morgenstern B, et al. Stability and inactivation of SARS coronavirus. Medical microbiology and immunology. 2005;194(1-2):1-6.

32. Lin TH, Tang FC, Hung PC, Hua ZC, Lai CY. Relative survival of Bacillus subtilis spores loaded on filtering facepiece respirators after five decontamination methods. Indoor air. 2018;28(5):754-762.

33. Rutala WA and Weber DJ. Guideline for Disinfection and Sterilization in Healthcare Facilities, 2008.

34. Swenson VA, Stacy AD, Gaylor MO, et al. Assessment and verification of commercially available pressure cookers for laboratory sterilization. PloS One. 2018;13(12).

35. Worley, S.D., D. Williams, and R.A. Crawford, Halamine water disinfectants. Critical Reviews in Environmental Science and Technology, 1988. 18(2): p. 133-175.

36. Prabhu S, Poulose EK. Silver nanoparticles: mechanism of antimicrobial action, synthesis, medical applications, and toxicity effects. International nano letters. 2012;2(1):32.

37. Kenawy E-R, Worley S, Broughton R. The chemistry and applications of antimicrobial polymers: a state-of-the-art review. Biomacromolecules. 2007;8(5):1359-1384.

38. van Doremalen N, Bushmaker T, Morris DH, et al. Aerosol and surface stability of SARS-CoV-2 as compared with SARS-CoV-1. New England Journal of Medicine. 2020.

39. Health Canada. Notice - Important Regulatory Considerations for the Reprocessing of Single Use N95 Respirators during the COVID-19 Response. 2020; https://www.canada.ca/en/health-canada/services/drugs-health-products/medicaldevices/activities/announcements/covid19-notice-reprocessing-n95-respirators.html. [Accessed April 27, 2020]

40. Kumar A, Kasloff SB, Leung A, et al. N95 Mask Decontamination using Standard Hospital Sterilization Technologies. medRxiv. 2020.

41. Bałazy A, Toivola M, Adhikari A, et al. Do N95 respirators provide $95 \%$ protection level against airborne viruses, and how adequate are surgical masks? American Journal of Infection Control. 2006;34(2):51-57. 
42. Fischer R, Morris DH, van Doremalen N, et al. Assessment of N95 respirator decontamination and re-use for SARS-CoV-2. medRxiv. 2020.

43. Elliott JH, Turner T, Clavisi O, et al. Living systematic reviews: an emerging opportunity to narrow the evidence-practice gap. PLoS Medicine. 2014;11(2):e1001603-e1001603.

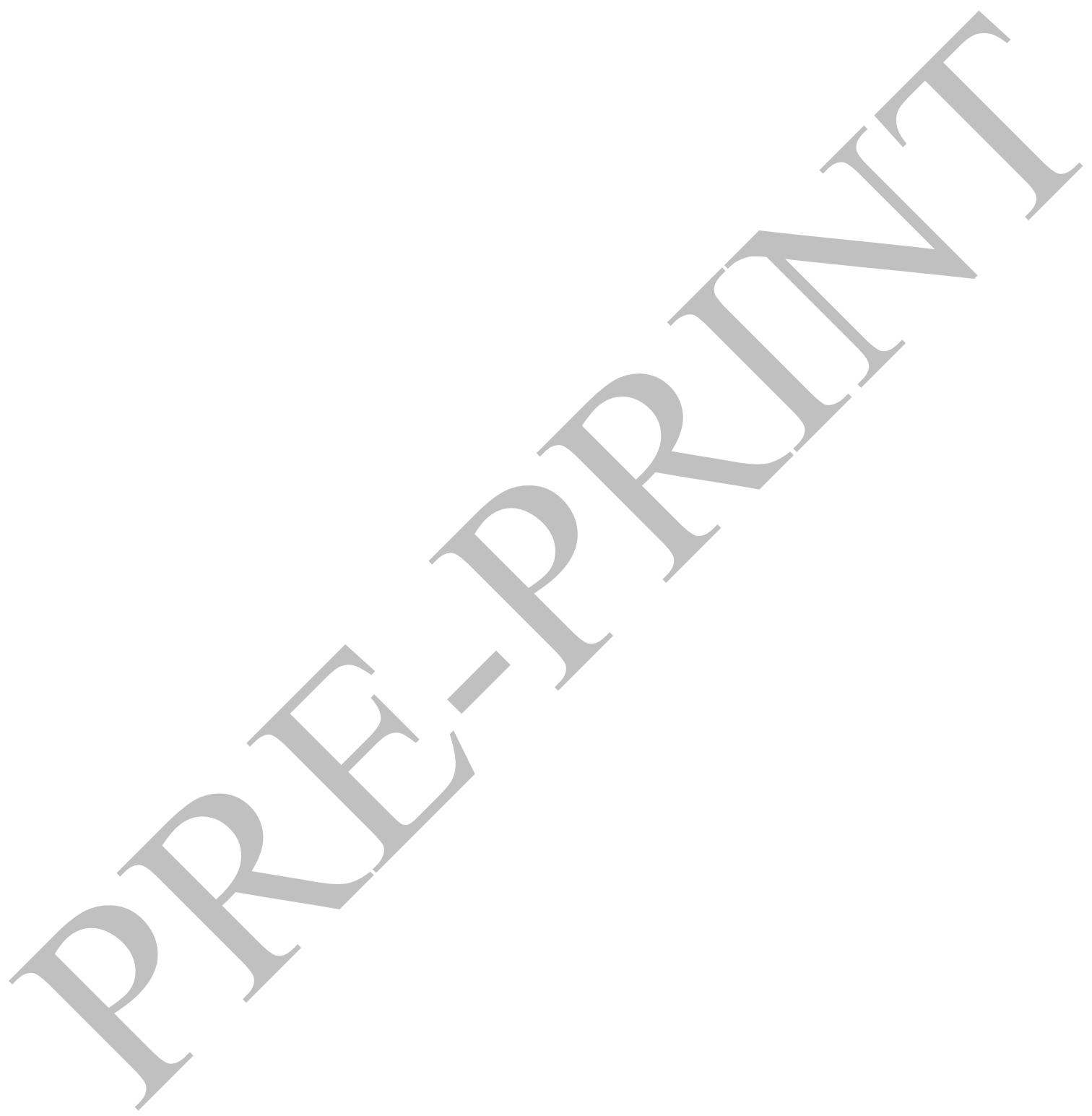


Figure 1. PRISMA Flow Diagram

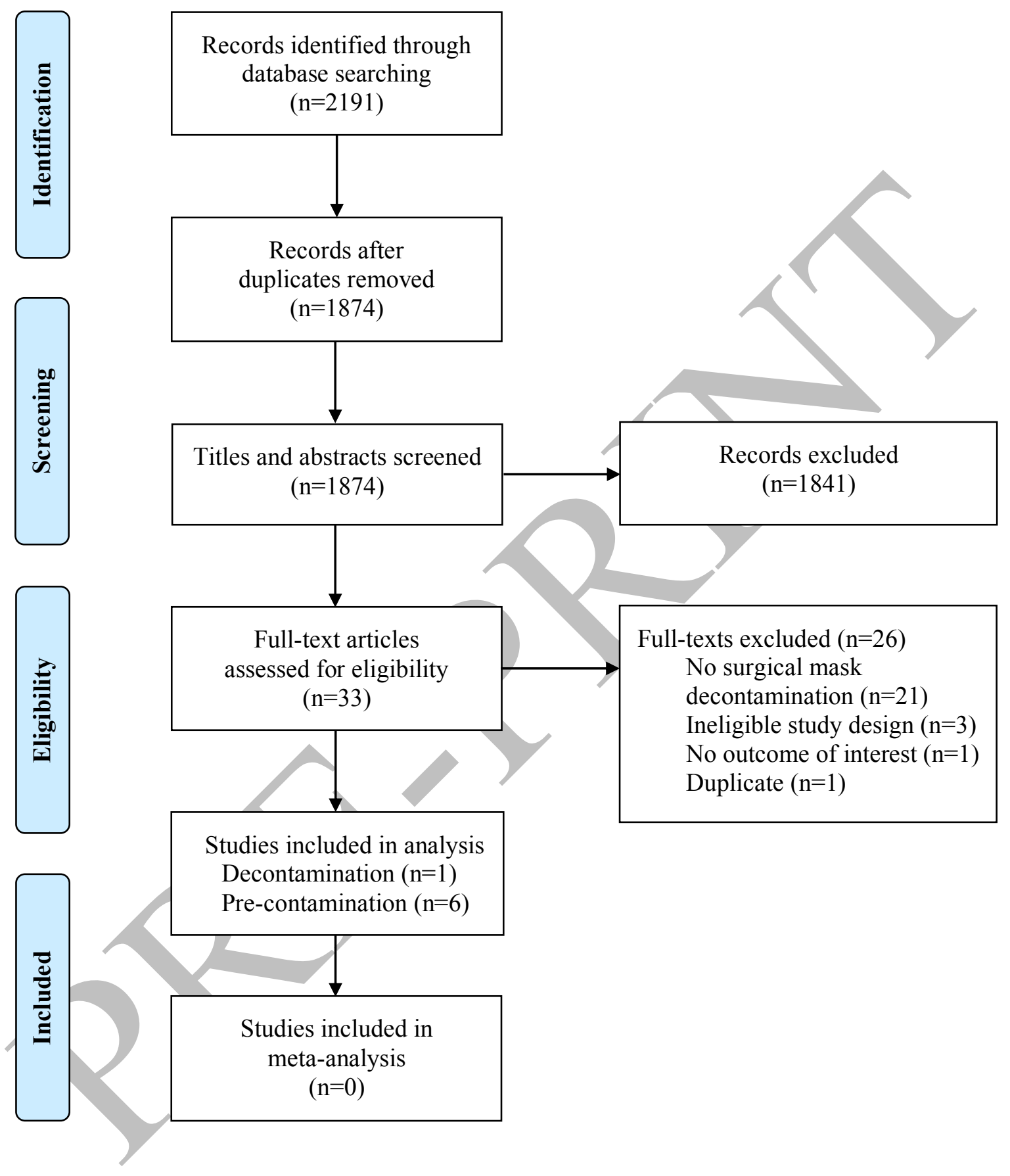


Table 1. Characteristics of included studies

\begin{tabular}{|c|c|c|c|c|c|c|}
\hline \multirow{2}{*}{$\begin{array}{c}\text { Author } \\
\text { (Year) }\end{array}$} & \multirow{2}{*}{ Intervention } & \multicolumn{2}{|c|}{ Procedure } & \multirow{2}{*}{$\begin{array}{c}\text { Mask/Component } \\
\text { Tested } \\
\end{array}$} & \multirow{2}{*}{ Testing Agent } & \multirow{2}{*}{ Control } \\
\hline & & Duration & Temperature & & & \\
\hline \multicolumn{7}{|c|}{ Decontamination Interventions } \\
\hline \multirow{5}{*}{$\underset{\text { (2017) }}{\text { Lin }}$} & Dry heat (rice cooker) & $3 \mathrm{~min}$ & $149-164{ }_{0} \mathrm{C}$ & \multirow{5}{*}{$\begin{array}{l}\text { Mask pieces: } \\
\text { 1. Gauze double-layer electret mask } \\
\text { 2. Oimo spunlace nonwoven masks } \\
\text { (models not specified) }\end{array}$} & \multirow{5}{*}{$\begin{array}{l}\text { Potassium sodium tartrate } \\
\text { tetrahydrate solution } \\
\text { (polydispersed droplets) }\end{array}$} & \multirow{5}{*}{$\begin{array}{l}\text { Untreated } \\
\text { mask piece }\end{array}$} \\
\hline & Moist heat (autoclave) & $15 \mathrm{~min}$ & $\begin{array}{c}\text { Pressure } \\
1.06 \mathrm{~kg} / \mathrm{cm} 2 \\
\end{array}$ & & & \\
\hline & Ethanol, $70 \%$ & $\begin{array}{c}10 \text { min } \\
\text { submersion }\end{array}$ & $\begin{array}{l}\text { Air dried } \\
\text { overnight }\end{array}$ & & & \\
\hline & Isopropanol, $100 \%$ & $\begin{array}{c}10 \text { min } \\
\text { submersion }\end{array}$ & $\begin{array}{l}\text { Air dried } \\
\text { overnight }\end{array}$ & & & \\
\hline & $\begin{array}{c}\text { Sodium hypochlorite } \\
\text { solution, } 0.5 \%\end{array}$ & $\begin{array}{c}10 \text { min } \\
\text { submersion }\end{array}$ & $\begin{array}{l}\text { Air dried } \\
\text { overnight }\end{array}$ & & & \\
\hline \multicolumn{7}{|c|}{ Pre-Contamination Interventions } \\
\hline $\begin{array}{c}\text { Quan } \\
\text { (2017) [23] }\end{array}$ & $\begin{array}{l}\text { Salt }(\mathrm{NaCl}) \text { coating: } \\
3,7,11,14 \text {, and } 19 \mathrm{mg} \\
\mathrm{NaCl} / \mathrm{cm}^{2}\end{array}$ & $\begin{array}{l}\text { Solution 1: } \\
\text { Overnight a } \\
\text { Solution 2: } \\
\text { 1-day a }\end{array}$ & $\begin{array}{l}\text { Oven-dried } \\
37 \mathrm{oC} \times 1 \text { day }\end{array}$ & $\begin{array}{l}\text { Salt-coated pieces of } \\
\text { polypropylene mask filter } \\
\text { (mask model not specified) }\end{array}$ & $\begin{array}{l}\text { Aerosolized virus: } \\
\text { H1N1 CA/09 } \\
\text { H1N1 PR/34 } \\
\text { H5N1 VN/04 }\end{array}$ & $\begin{array}{l}\text { Uncoated } \\
\text { mask filter }\end{array}$ \\
\hline $\begin{array}{c}\text { Tseng } \\
(2016)[25]\end{array}$ & $\begin{array}{l}\text { Quaternary ammonium } \\
\text { agent (Goldshield 5; } \\
\text { GS5), } 1 \% \text { in sterile } \\
\text { water }\end{array}$ & $\begin{array}{l}\text { Applied with common spray } \\
\text { bottle }(4 \text { sprays }=1 \mathrm{~mL})\end{array}$ & Room & $\begin{array}{l}\text { SS5-coated pieces of } 3 \text { mask layers } \\
\text { and GS5-coated full mask } \\
\text { (AERO PRO, Taiwan; } \\
\text { model not specified) }\end{array}$ & $\begin{array}{c}\text { Aerosolized bacteria: } \\
\text { A. baumannii ATCC } 17978 \\
\text { E. faecalis ATCC } 29212 \\
\text { S. aureus ATCC } 29213 \\
\end{array}$ & $\begin{array}{l}\text { Sterile water } \\
\text { coated mask }\end{array}$ \\
\hline $\begin{array}{l}\text { Demir } \\
(2015)[20]\end{array}$ & $\begin{array}{l}\text { N-halamine (1-chloro- } \\
2,2,5,5 \text { - tetramethyl-4- } \\
\text { imidazolidinone) } \\
1 \mathrm{wt} \% \text { in ethanol }\end{array}$ & $\begin{array}{c}10 \text { min } \\
\text { submersion } \\
\text { Padded through } \\
\text { laboratory wringer } \\
\end{array}$ & & $\begin{array}{l}\text { N-halamine-coated pieces of } \\
\text { polypropylene mask filter } \\
\text { (Hollingsworth \& Vose Company, } \\
\text { USA; model not specified) }\end{array}$ & $\begin{array}{c}\text { Aerosolized bacteria: } \\
\text { S. aureus ATCC6538 } \\
\text { E. coli } 0157: \mathrm{H} 7 \\
\text { ATCC } 43895\end{array}$ & $\begin{array}{l}\text { Uncoated } \\
\text { mask filter }\end{array}$ \\
\hline $\begin{array}{c}\mathrm{Li} \\
(2006-\mathrm{A})[22]\end{array}$ & \multirow{2}{*}{$\begin{array}{c}\text { Silver nitrate and } \\
\text { titanium dioxide } \\
\text { nanoparticle emulsion, } \\
0.4 \mathrm{mg} / \mathrm{cm}^{2}\end{array}$} & \multirow{2}{*}{$\begin{array}{r}\text { Coated to desir } \\
\text { textile-fir } \\
\text { (China p }\end{array}$} & d concentration with & $\begin{array}{c}\text { Full masks with nanoparticle-coated } \\
\text { outermost mask layer } \\
\text { (Winner Medical Group, China; } \\
\text { model not specified) }\end{array}$ & $\begin{array}{c}\text { Aerosolized pathogen } \\
\text { simulant: KCl-fluorescein }\end{array}$ & $\begin{array}{l}\text { Uncoated } \\
\text { mask layer }\end{array}$ \\
\hline $\begin{array}{c}\mathrm{Li} \\
(2006-\mathrm{B})^{[21]}\end{array}$ & & & ent 03142467) & $\begin{array}{c}\text { Nanoparticle-coated pieces of } \\
\text { outermost mask layer } \\
\text { (Winner Medical Group, China; } \\
\text { model not specified) }\end{array}$ & $\begin{array}{l}\text { Bacterial suspensions: } \\
\text { E. coli ATCC25922 } \\
\text { S. aureus ATCC25923 }\end{array}$ & $\begin{array}{l}\text { Uncoated } \\
\text { fabric }\end{array}$ \\
\hline $\begin{array}{c}\text { Shen } \\
(2005)[24]\end{array}$ & $\begin{array}{c}\text { Fluorochemical } \\
\text { repellent (Zonyl® PPR } \\
\text { Protector), } 6 \% \text { and 12\% }\end{array}$ & $\begin{array}{c}\text { Unspecified } \\
\text { submersion time } \\
\text { Padded through } \\
\text { laboratory wringer }\end{array}$ & $\begin{array}{l}\text { Dried at } 176_{0} \mathrm{~F} \\
\quad \times 2 \min \\
\text { Cured at } 250_{0} \mathrm{~F} \\
\times 2 \min \\
\end{array}$ & $\begin{array}{l}3 \text { full masks made of nonwoven } \\
\text { mask components with repellant } \\
\text { applied to cover layer } \\
\text { (models not specified) }\end{array}$ & $\begin{array}{l}\text { Aerosolized pathogen } \\
\text { simulant: Latex micro- } \\
\text { spheres + synthetic blood }\end{array}$ & $\begin{array}{l}\text { Uncoated } \\
\text { mask }\end{array}$ \\
\hline
\end{tabular}

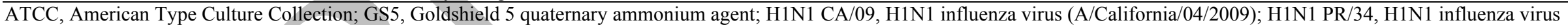

(A/Puerto Rico/08/2934); H5N1 VN/04, H5N1 influenza virus (A/Vietnam/1203/2004); KCl, potassium chloride; NaCl, sodium chloride.

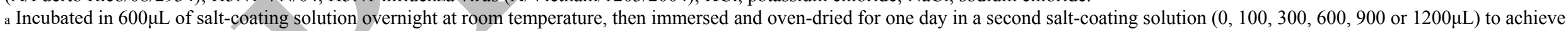
different salt-coating (sodium chloride, in $\mathrm{mg}$ ) on filter per unit area. 
Table 2. Decontamination methods and summary of results by outcome (Lin et al., 2017) [19]

$\begin{array}{ccc}\text { Test Agent \& Conditions } & \text { Intervention } & \begin{array}{c}\text { Mask/ } \\ \text { Component }\end{array}\end{array}$

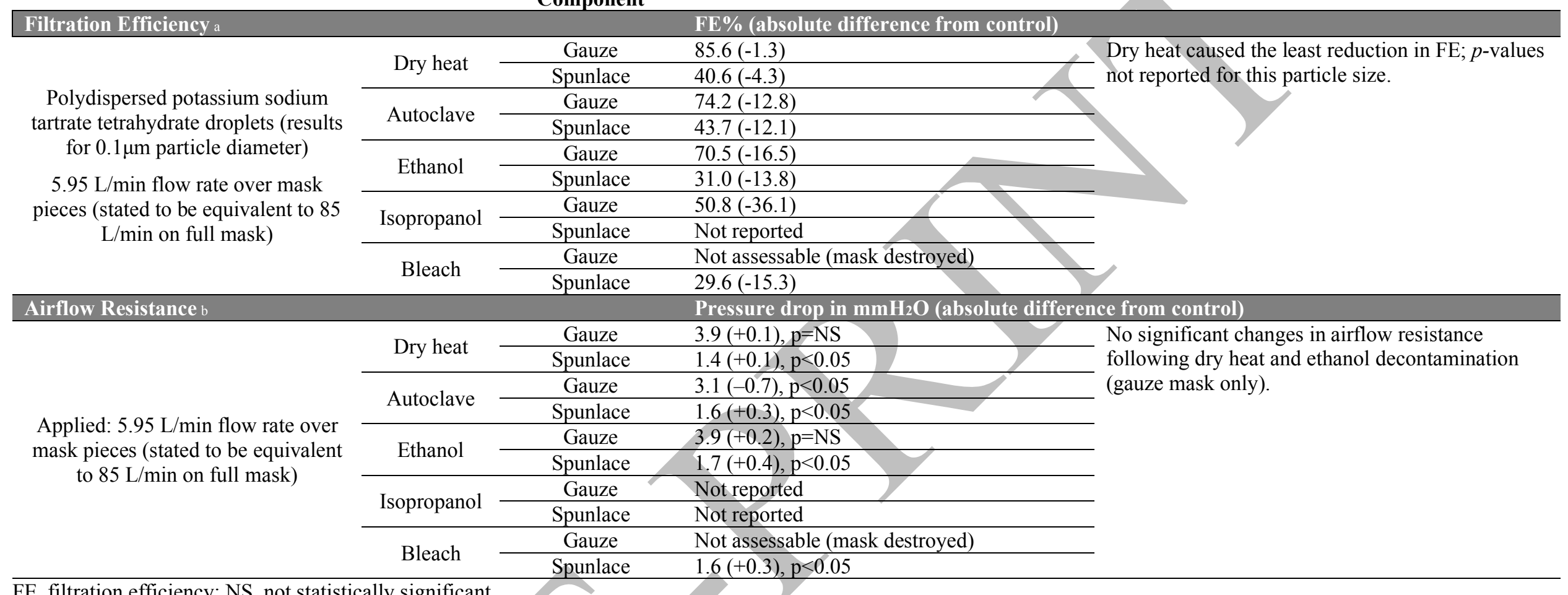

FE, filtration efficiency; NS, not statistically significant.

a FE to testing agent used, expressed as a percentage. A higher percentage filtration efficiency indicates better mask performance. Results in study were presented as percentage particle penetration, and converted to filtration efficiency ( $\mathrm{FE} \%=100$ - particle penetration) for consistency of reporting in this systematic review.

b Airflow resistance assessed the "breathability" of the mask at tidal breathing. A lower airflow resistance means better breathability.

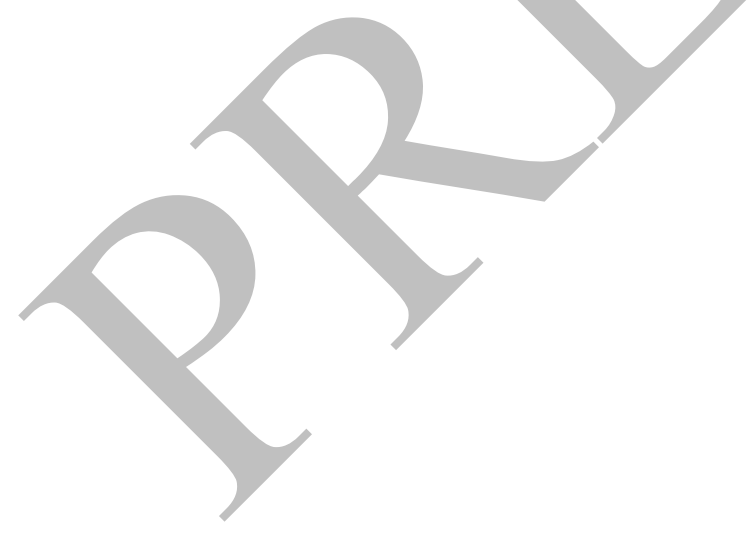


Table 3. Pre-contamination methods and summary of results by outcome

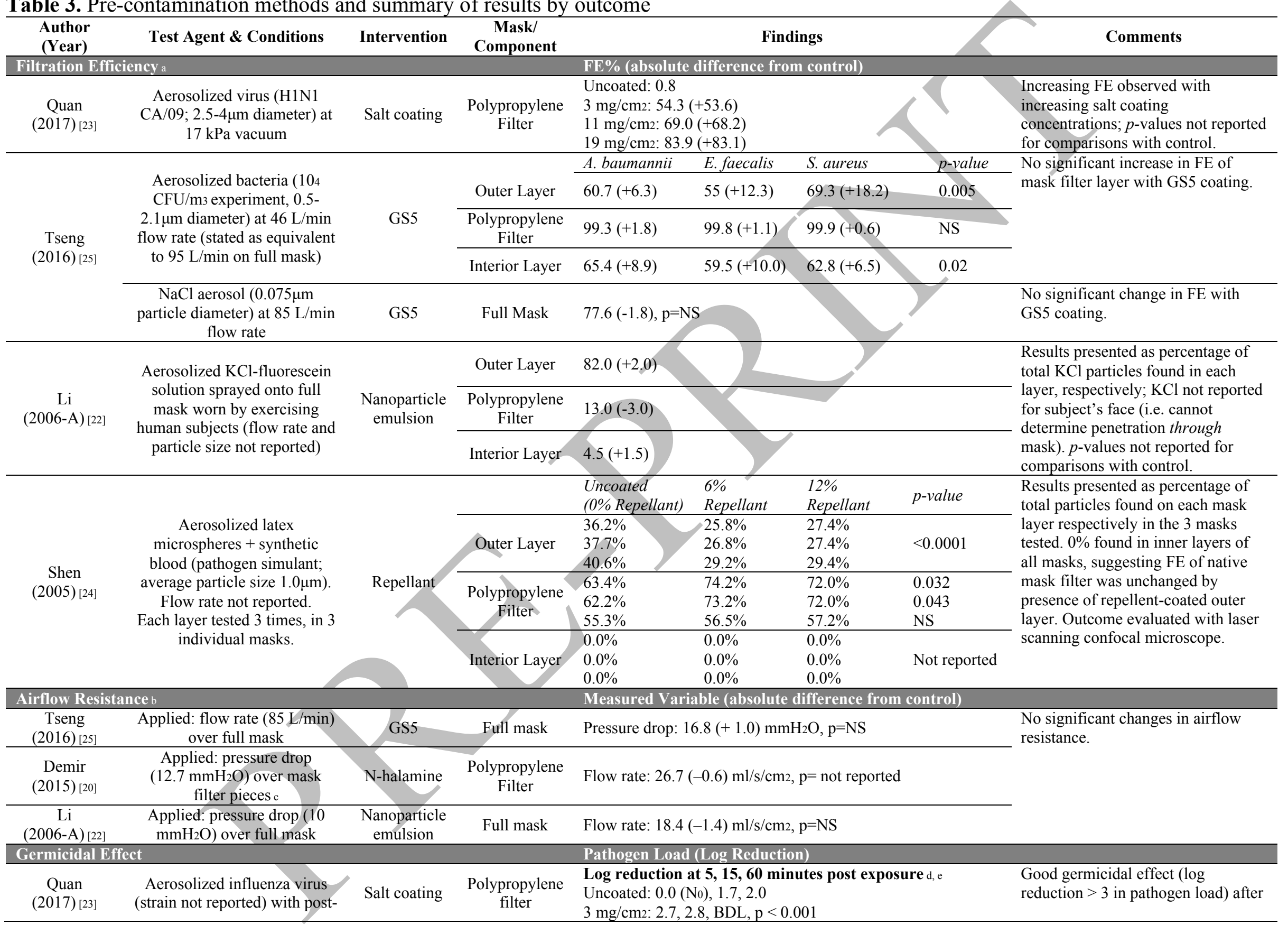




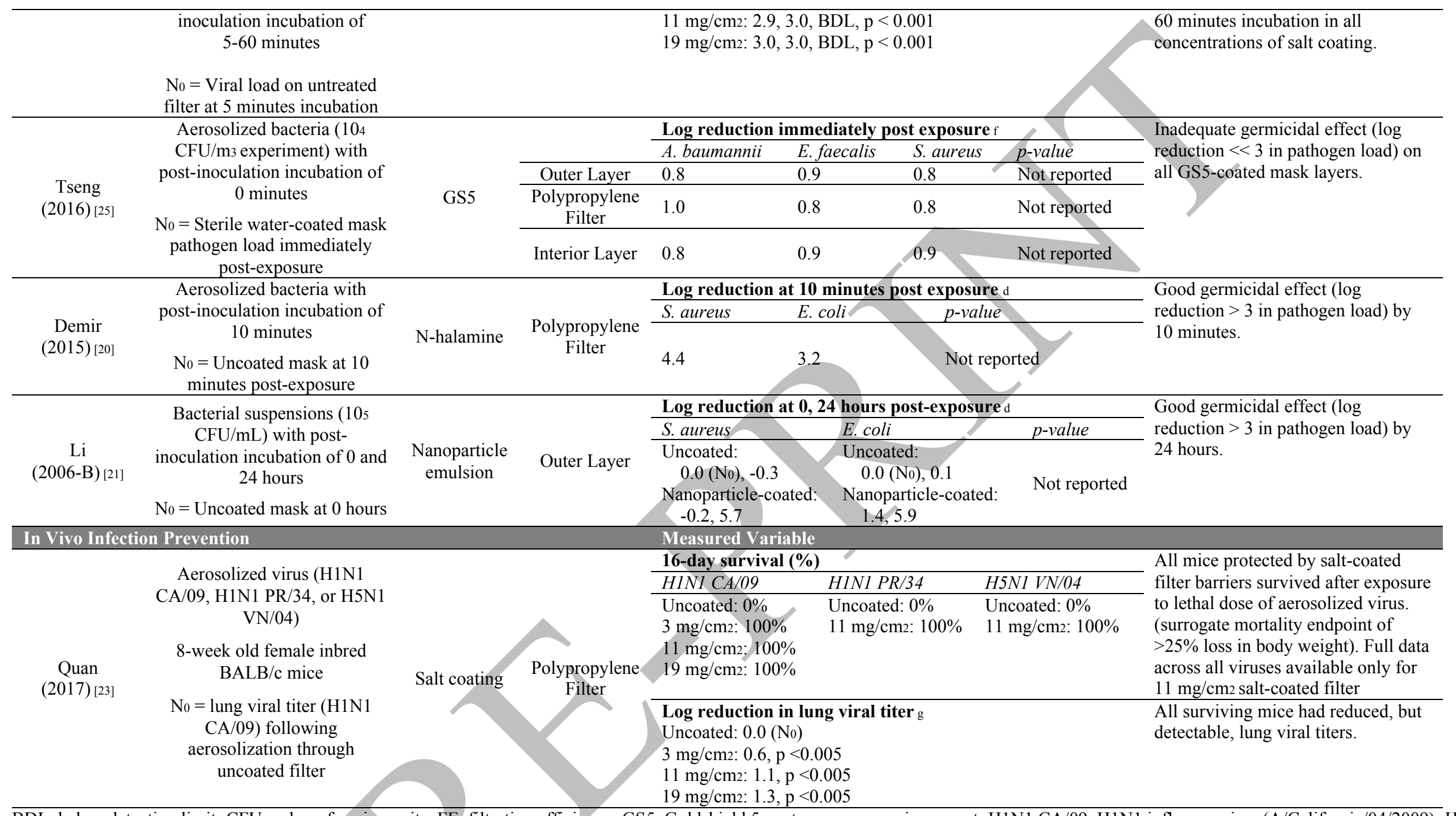

$\overline{\mathrm{BDL}}$, below detection limit; CFU, colony forming units; FE, filtration efficiency; GS5, Goldshield 5 quaternary ammonium agent; H1N1 CA/09, H1N1 influenza virus (A/California/04/2009); H1N1 $\mathrm{PR} / 34, \mathrm{H} 1 \mathrm{~N} 1$ influenza virus (A/Puerto Rico/08/2934); H5N1 VN/04, H5N1 influenza virus (A/Vietnam/1203/2004); KCl, potassium chloride; No, time zero from which log reduction was calculated; $\mathrm{NaCl}$, sodium chloride; NS, not statistically significant.

a FE to testing agent used, expressed as a percentage. A higher percentage filtration efficiency indicates better mask performance.

b Airflow resistance assessed the "breathability" of the mask at tidal breathing. A lower airflow resistance means better breathability.

c Study reported pressure drop and flow rate in inches of water and cubic feet per minute per square foot, respectively. Results converted to SI units.

d Colony-forming units or plaque-forming units reported in study, as applicable. Results converted to log reductions.

e Plaque-forming units below detectable limit. Detection limit of assay not reported; log reduction cannot be calculated.

f Colony-forming unit reduction percentages reported in study. Results converted to log reductions.

$\mathrm{g}$ Absolute values for lung viral titers reported in stûdy. Results converted to log reductions. 